Supporting Information for:

\title{
Bioinspired Construction of Uranium Ion Trap with Abundant Phosphate
}

\section{Functional Groups}

Yaguang Peng ${ }^{*}, \dagger$, Yuxi Zhang ${ }^{\ddagger}$, Qiang Tan ${ }^{\ddagger}$, Hongliang Huang ${ }^{*}, \S$

'Department of Chemistry, Tsinghua University, Beijing 100084, China

${ }^{\ddagger}$ State Key Laboratory of Organic-Inorganic Composites, Beijing University of

Chemical Technology, Beijing 100029, China

${ }^{\S}$ State Key Laboratory of Separation Membranes and Membrane Processes, Tianjin Polytechnic University, Tianjin 300387, China

*Corresponding authors. E-mail address: pengyaguang@mail.tsinghua.edu.cn huanghongliang@tiangong.edu.cn 


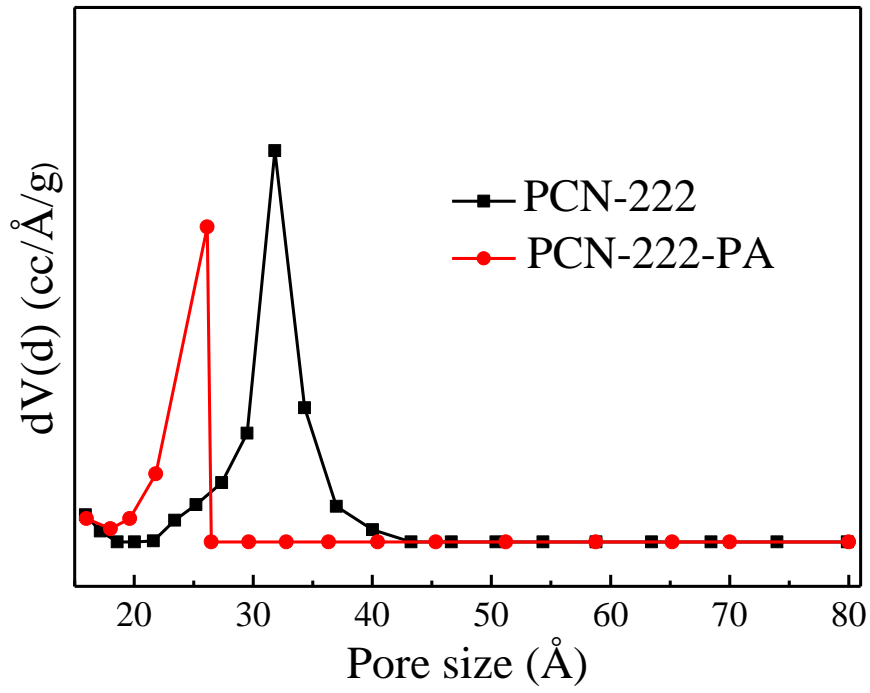

Figure S1 Pore size distribution of PCN-222 before and after PA modification.

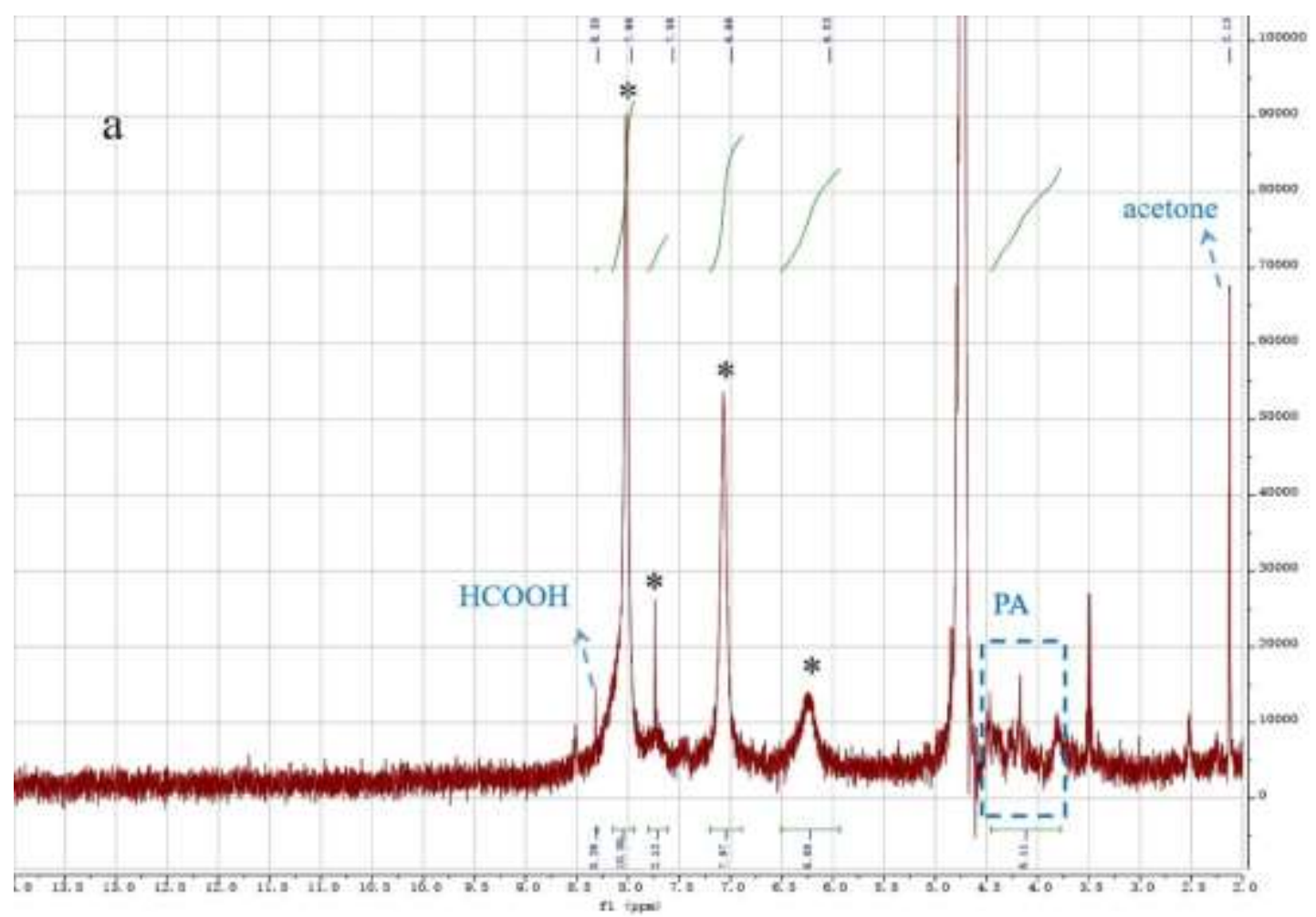




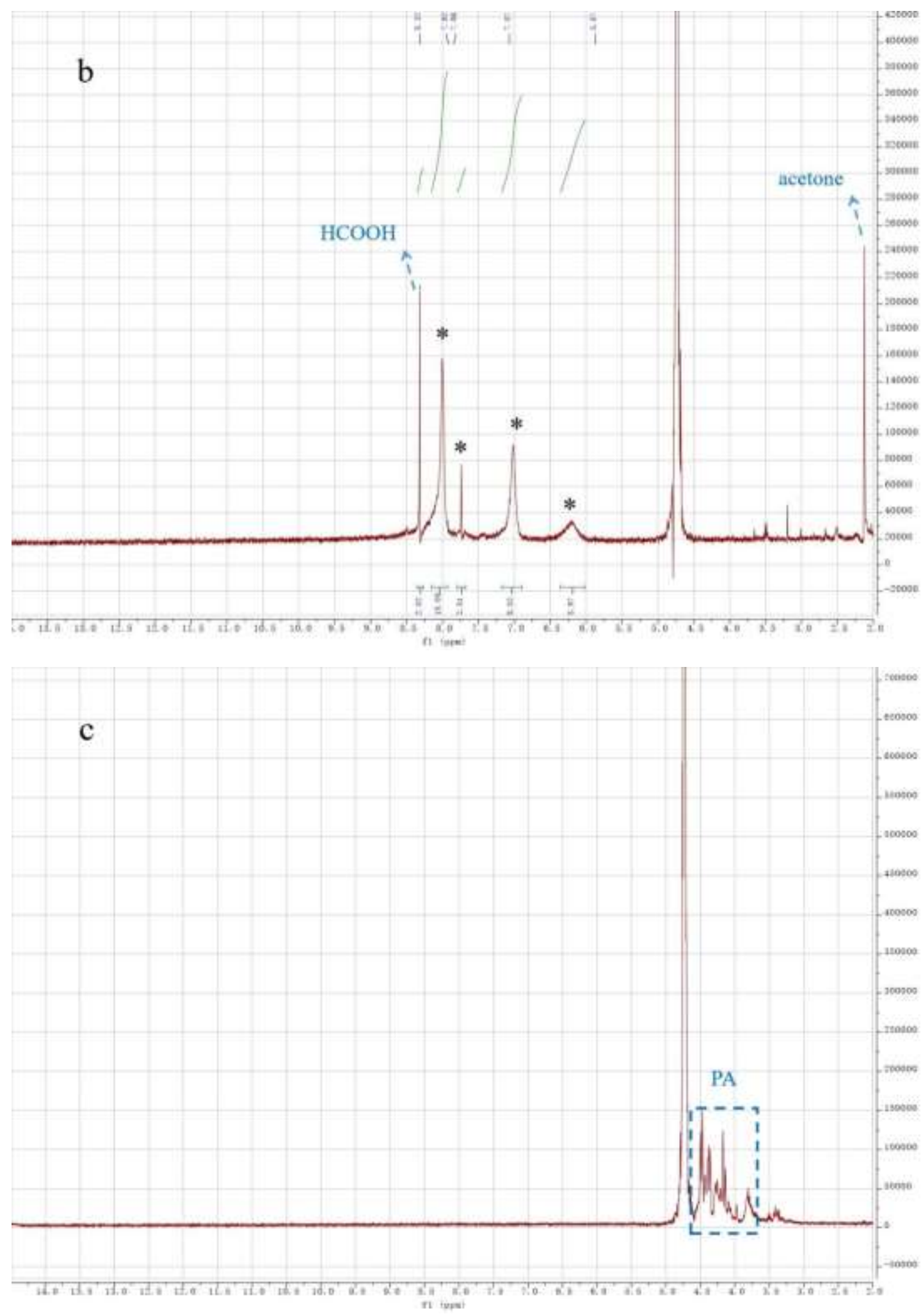

Figure S2 The ${ }^{1} \mathrm{H}$ NMR spectra of (a) digested PCN-222-PA, (b)digested PCN-222 and (c) PA in $\mathrm{KOH} / \mathrm{D}_{2} \mathrm{O}$. 


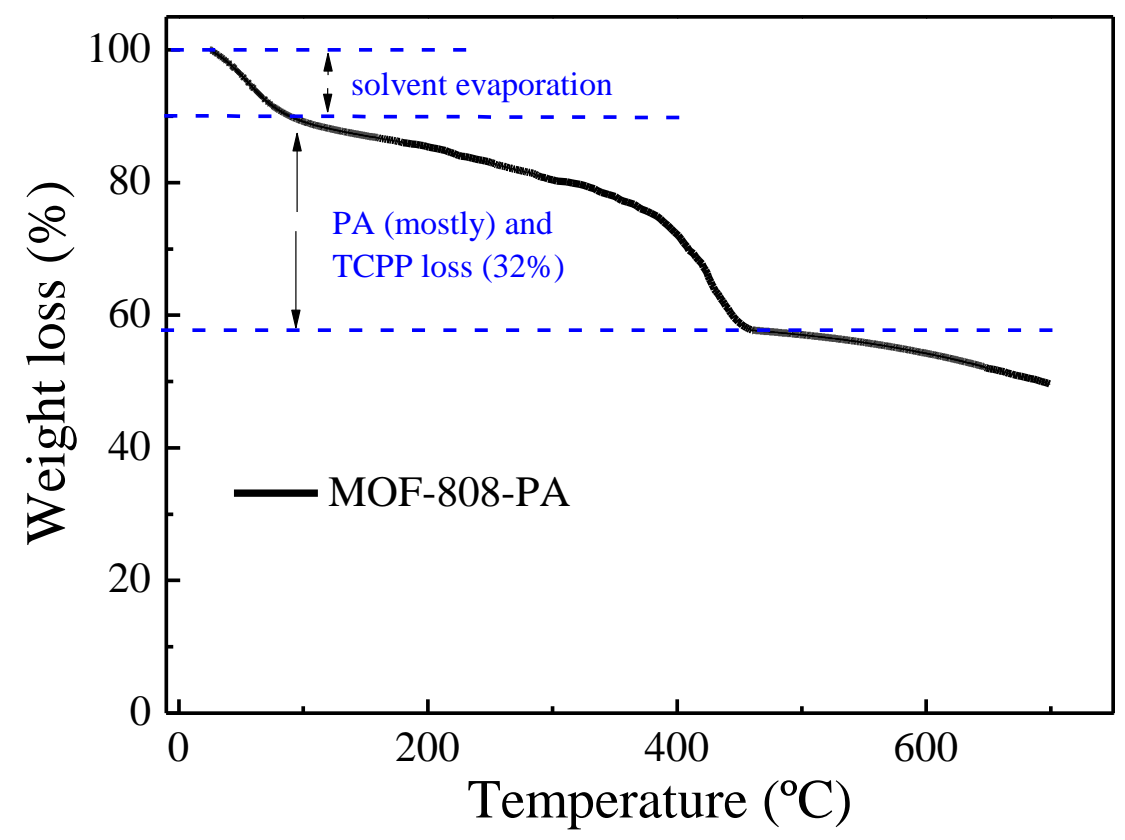

Figure S3 TGA plot of MOF-808-PA in $\mathrm{N}_{2}$ atmosphere.

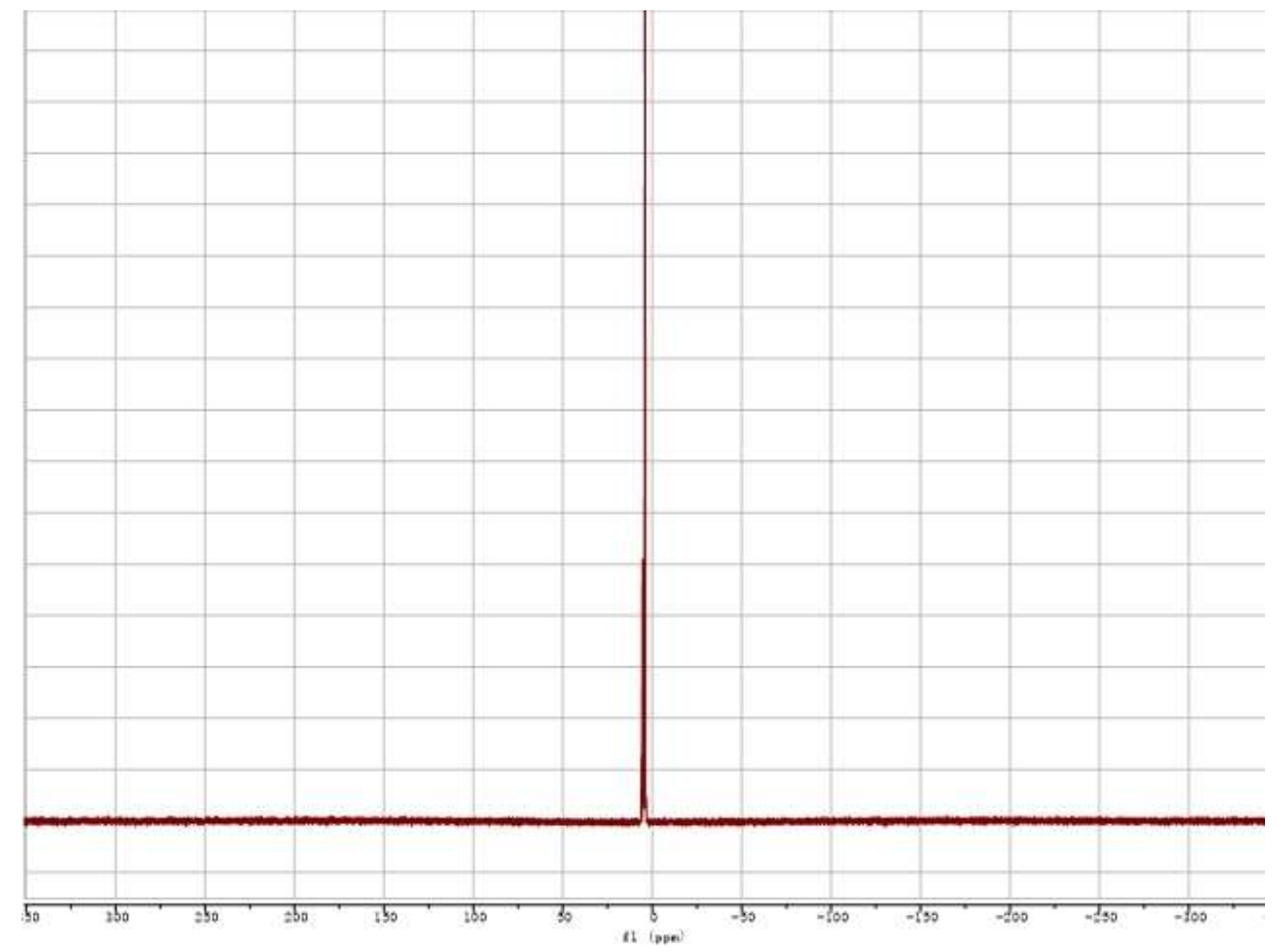

Figure S4 The ${ }^{31} \mathrm{P}$ NMR spectra of digested $\mathrm{PCN}-222-\mathrm{PA}$ in $\mathrm{KOH} / \mathrm{D}_{2} \mathrm{O}$ 


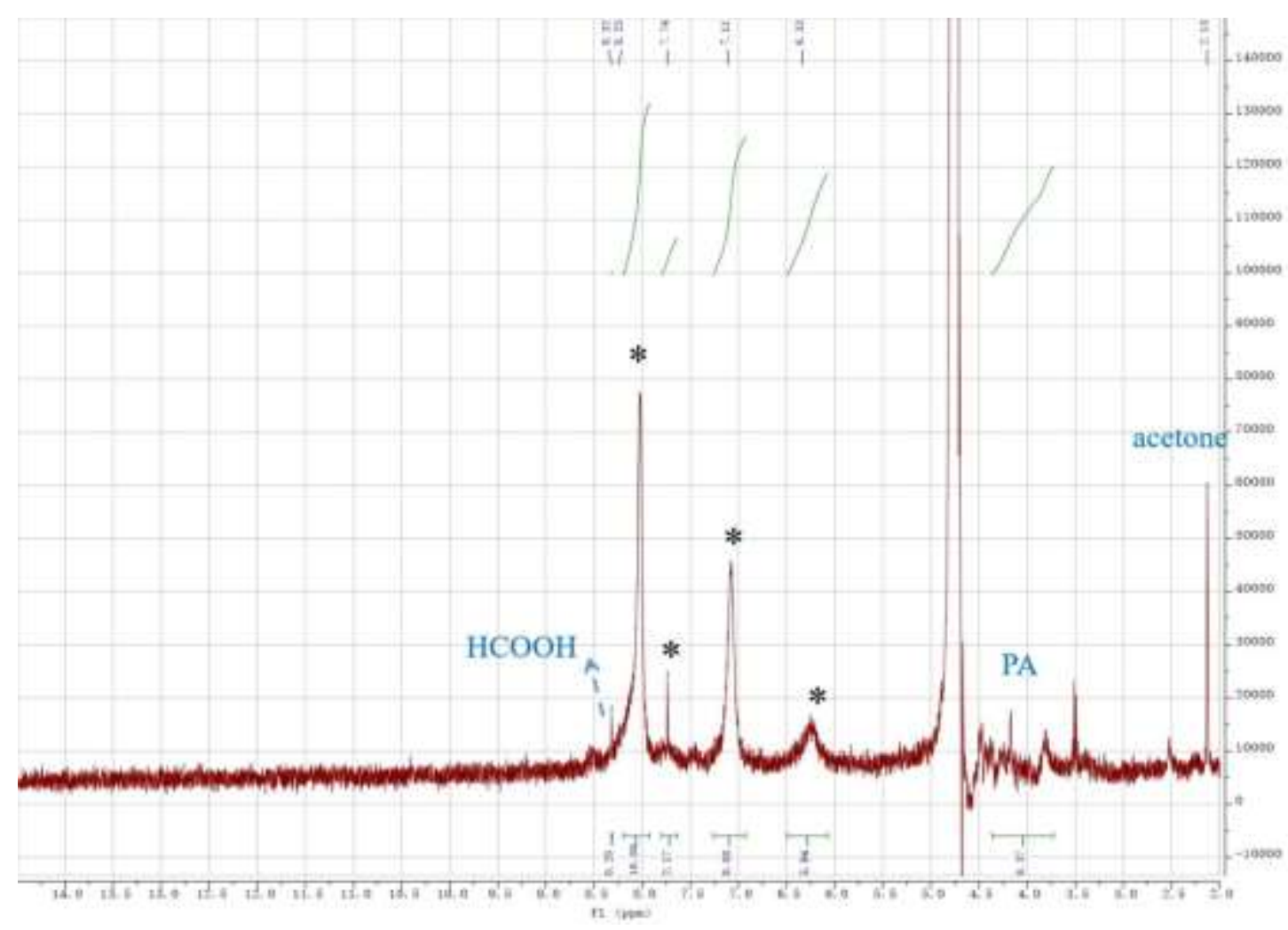

Figure S5 The ${ }^{1} \mathrm{H}$ NMR spectra of digested PCN-222-PA with the PA concentration of $5 \mathrm{mM}$.

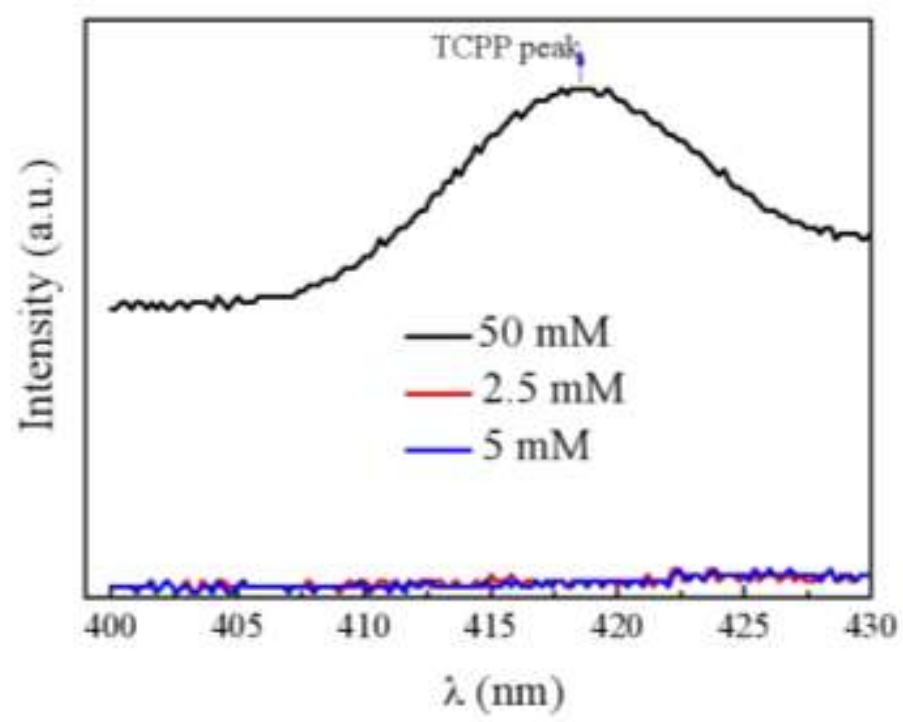

Figure S6 UV spectra of synthetic solution of PCN-222-PA at different concentrations. 


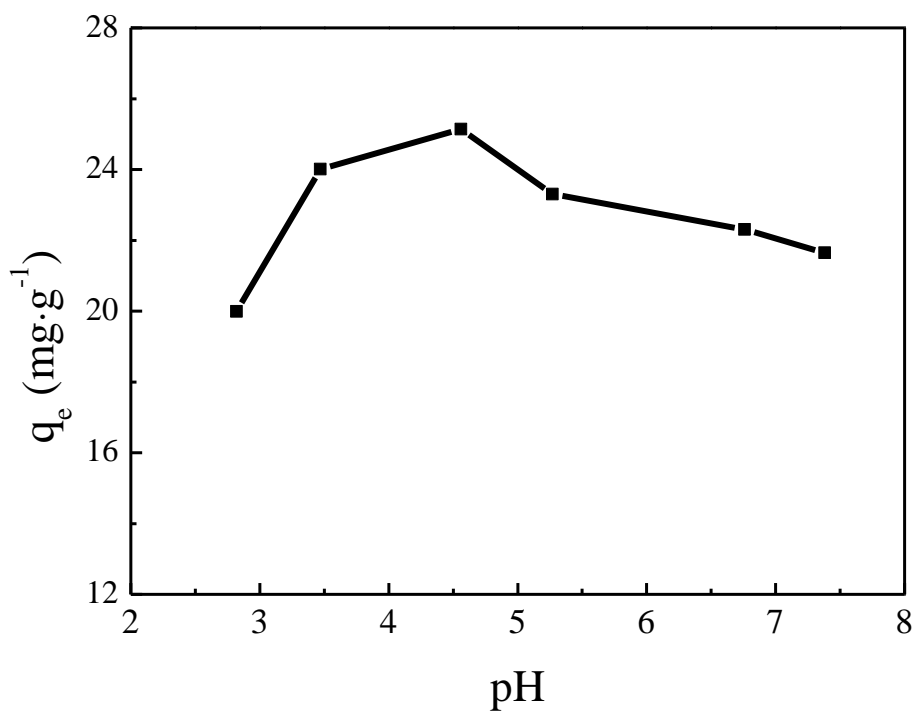

Figure S7 Effect of pH on U(VI) adsorption over PCN-222-PA.
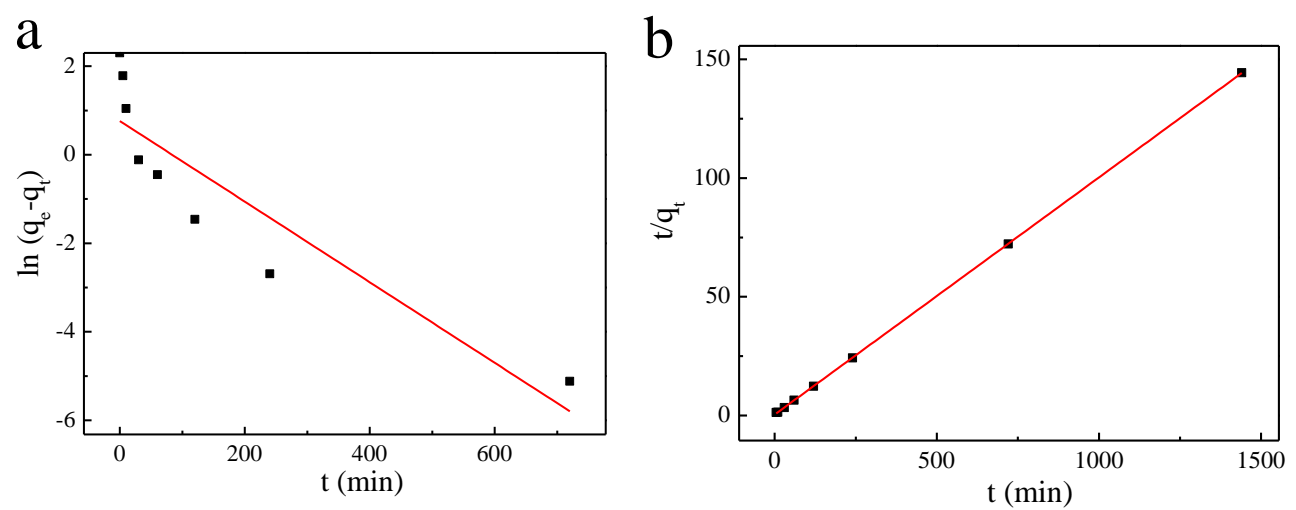

Figure S8 The linear fitting curves of (a) pseudo-first-order kinetic model and (b) pseudo-second-order kinetic model for PCN-222-PA toward U(VI). 


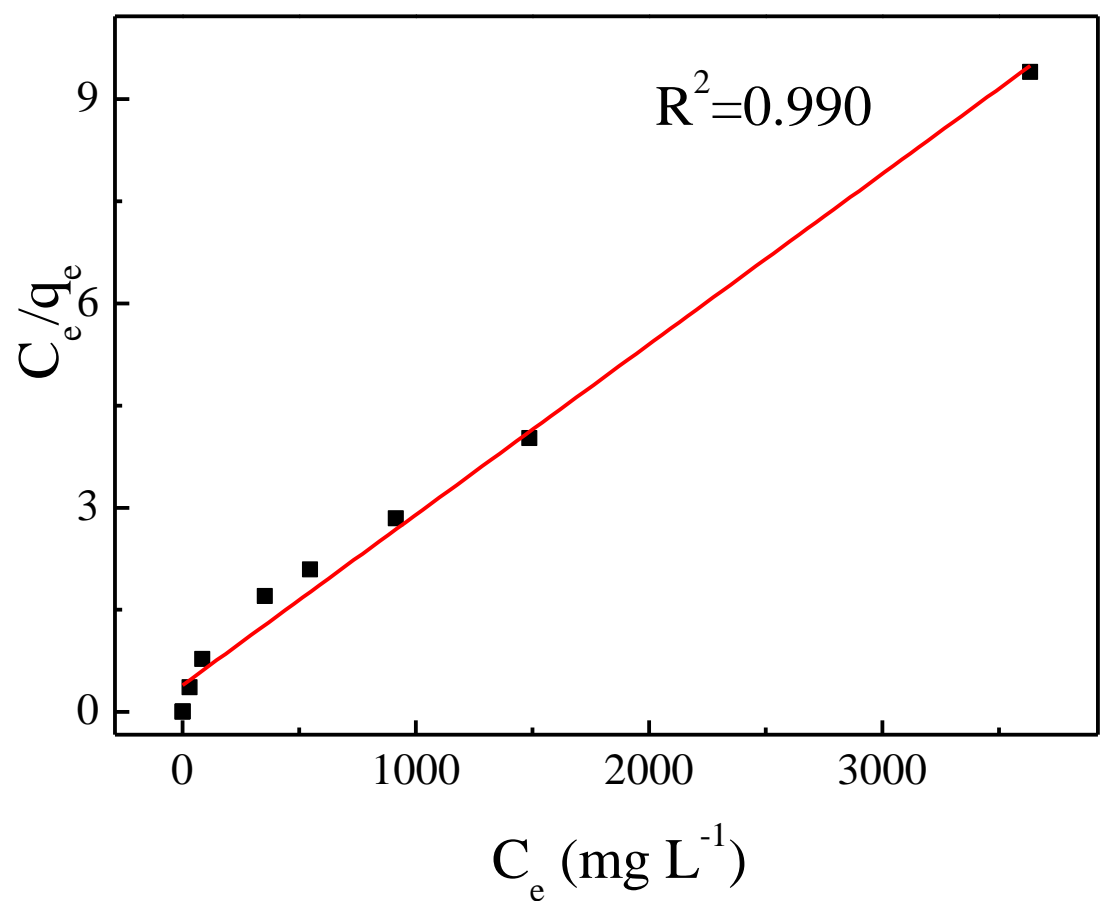

Figure S9 The linear fitting curves of Langmuir model for PCN-222-PA toward $\mathrm{U}(\mathrm{VI})$.

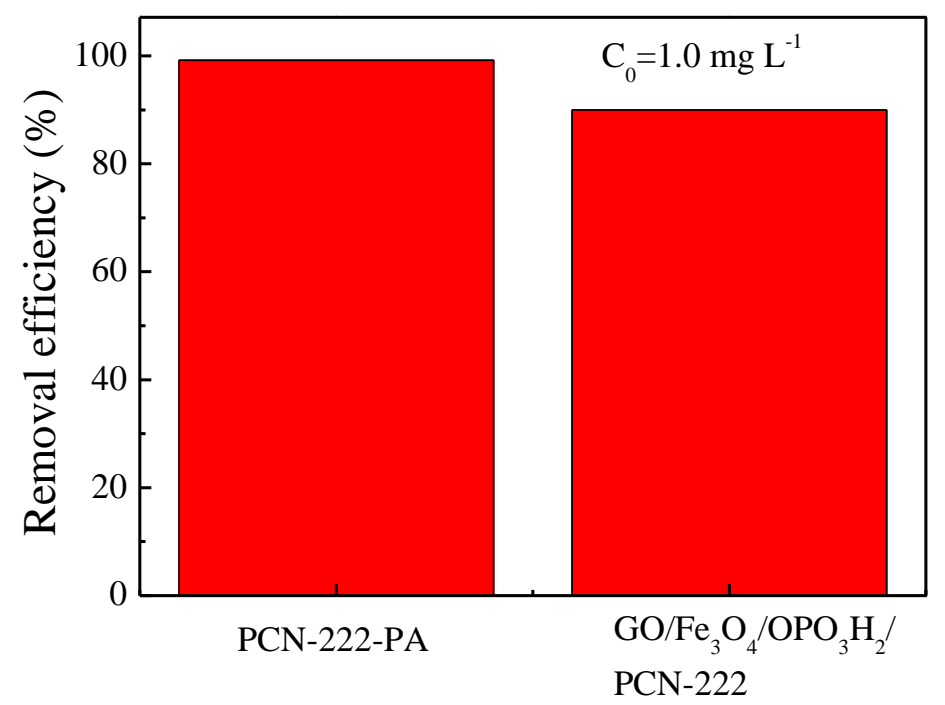

Figure S10 Removal efficiency of PCN-222-PA and $\mathrm{GO} / \mathrm{Fe}_{3} \mathrm{O}_{4} / \mathrm{OPO}_{3} \mathrm{H}_{2} / \mathrm{PCN}-222$ (J. Ind. Eng. Chem., 2021, 93, 322-332) toward U(VI) at the initial concentration of $1.0 \mathrm{mg} \mathrm{L}^{-1}$ 


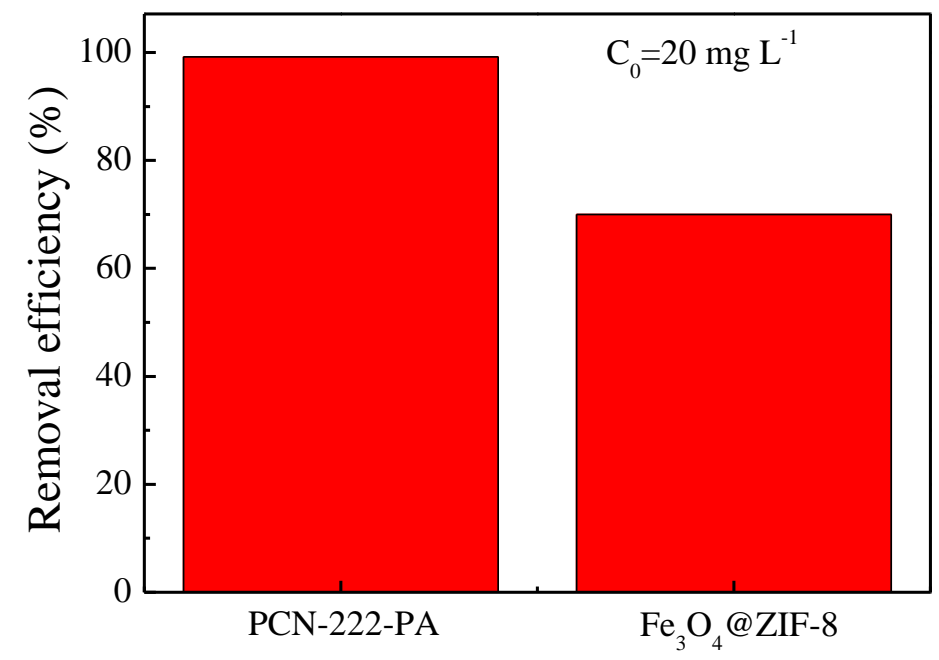

Figure S11 Removal efficiency of PCN-222-PA and Fe $\mathrm{O}_{4} @ \mathrm{ZIF}-8$ (Chem. Eng. J., $2019,378,122105)$ toward $\mathrm{U}(\mathrm{VI})$ at the initial concentration of $20 \mathrm{mg} \mathrm{L}^{-1}$.

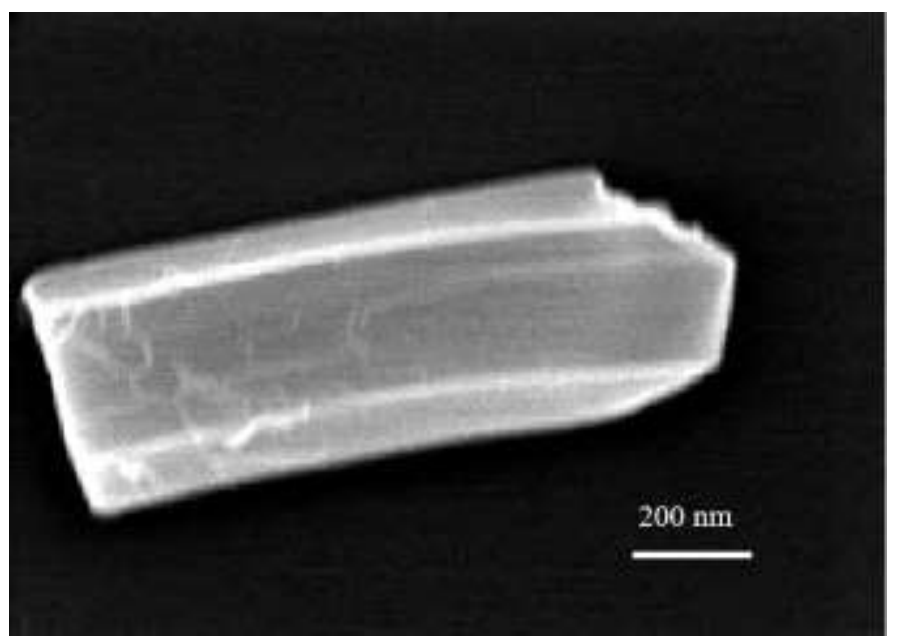

Figure S12 SEM image of PCN-222-PA (small size).
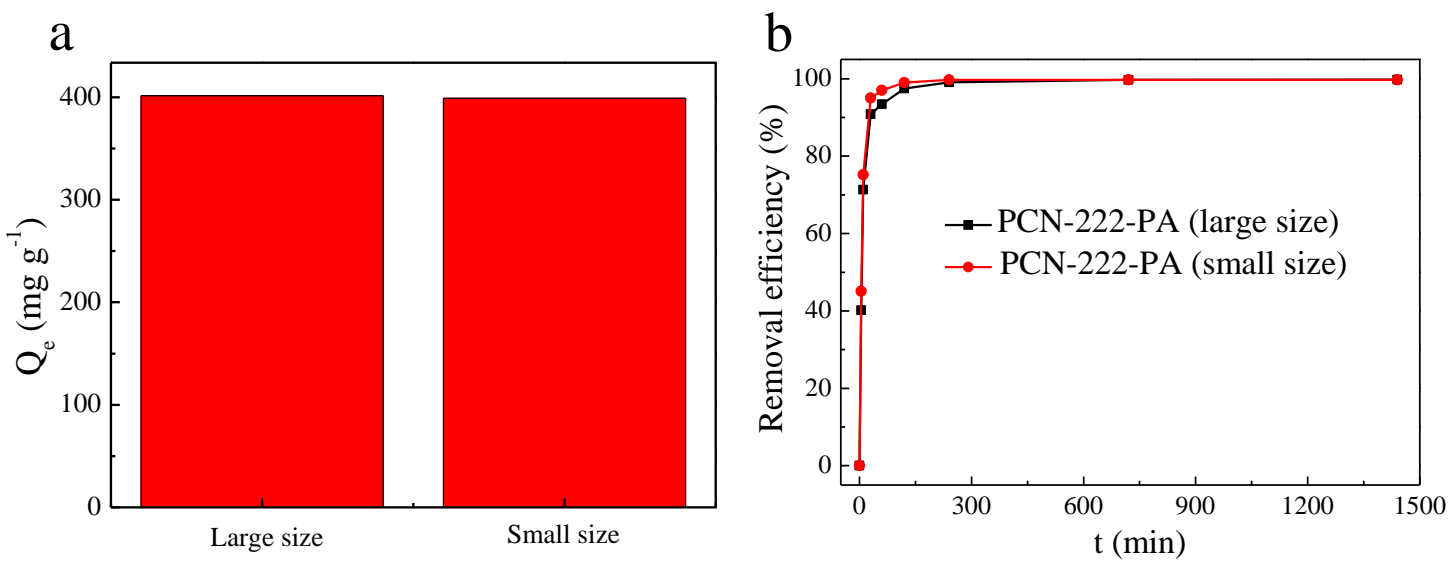

Figure S13 (a) U(VI) maximum uptake capacity and (b) adsorption kinetics of 
PCN-222-PA with different crystal sizes.

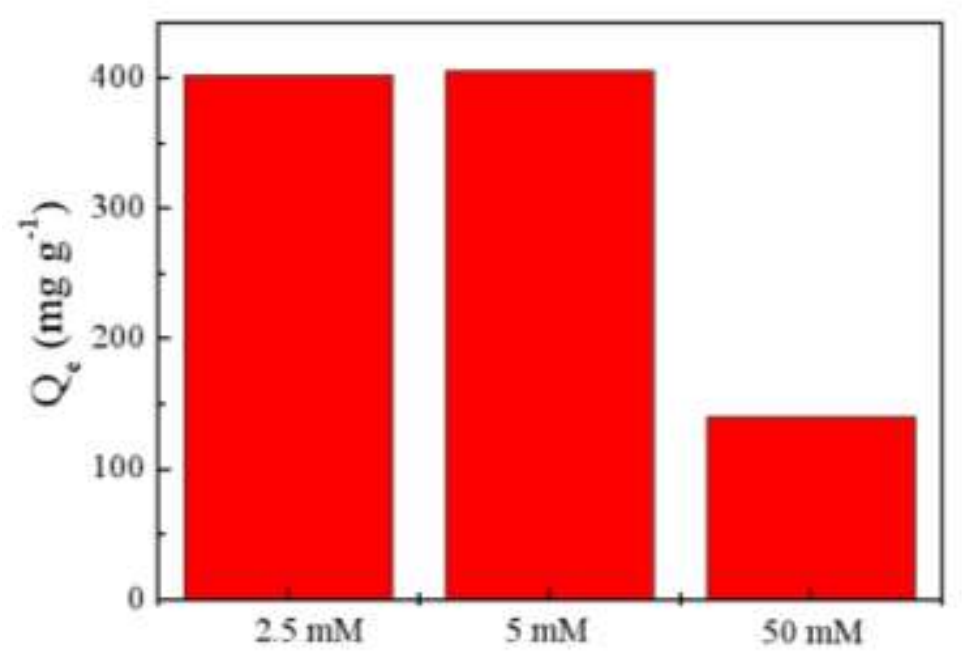

Figure S14 The effect of PA initial concentration on U(VI) maximum adsorption capacity.

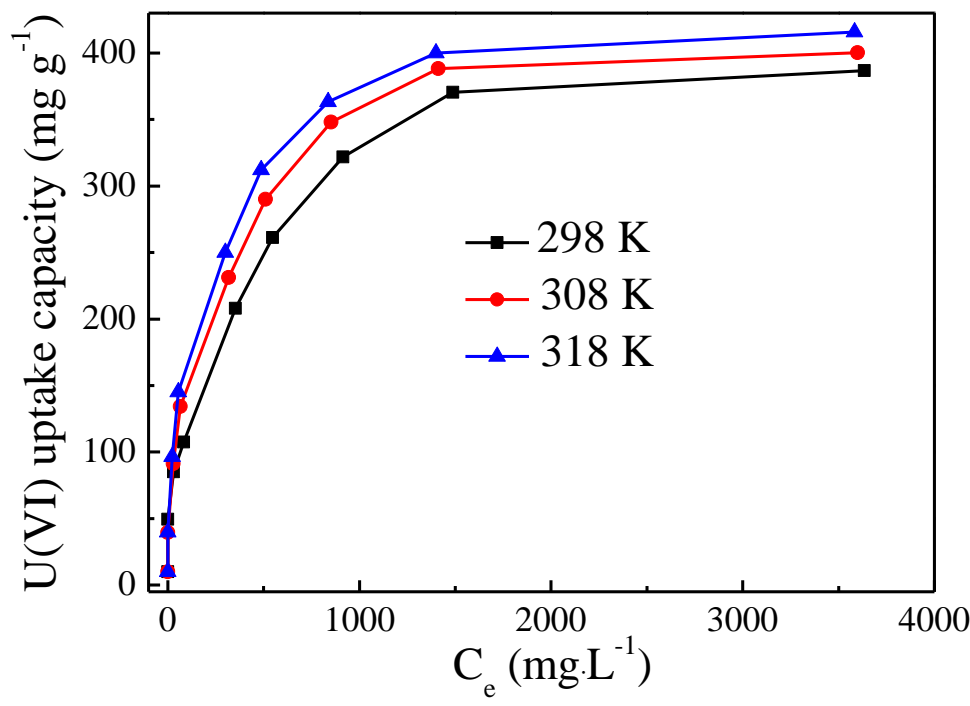

Figure S15 The adsorption isotherms of PCN-222-PA toward U(VI) with different temperatures. 


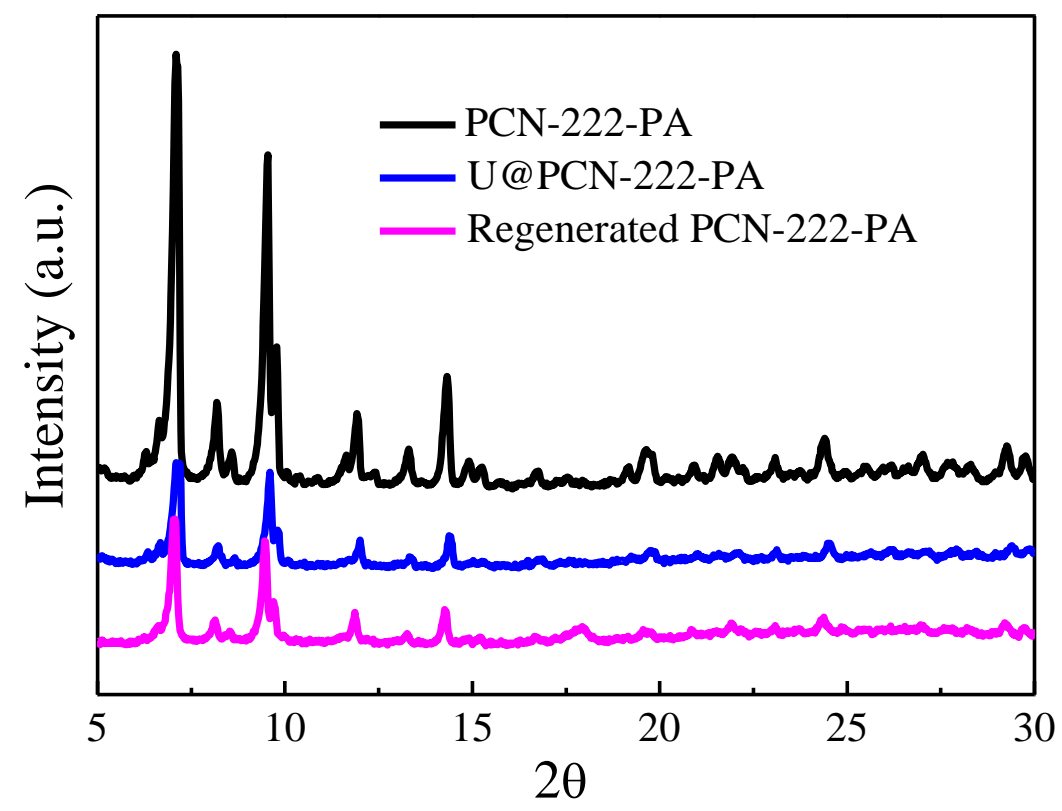

Figure S16 PXRD patterns of PCN-222-PA before and after U (VI) adsorption and regeneration.

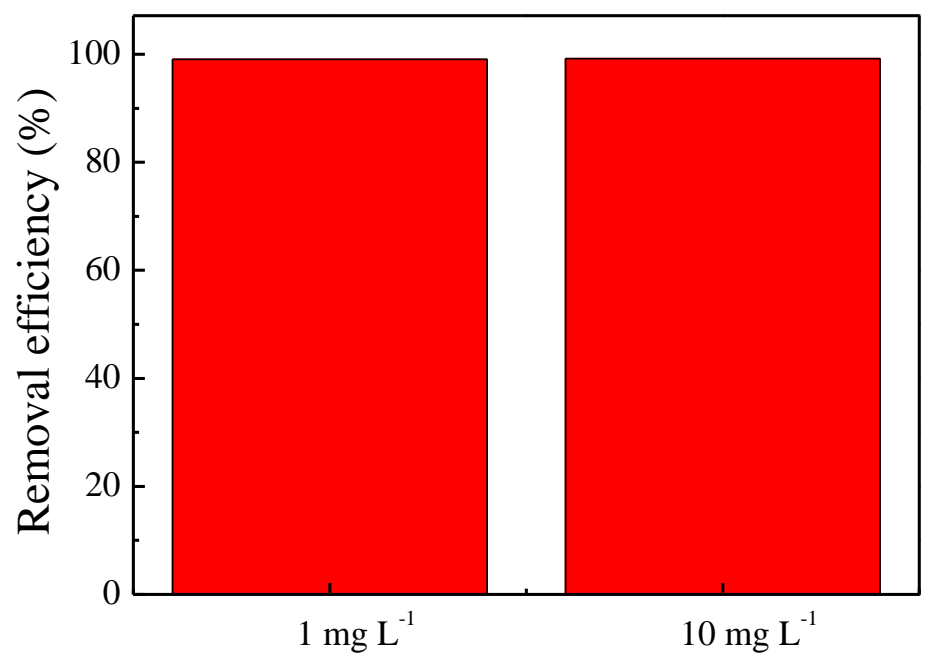

Figure S17 Removal efficiency of PCN-222-PA toward U(VI) with different 
concentrations $(\mathrm{pH}=8)$.

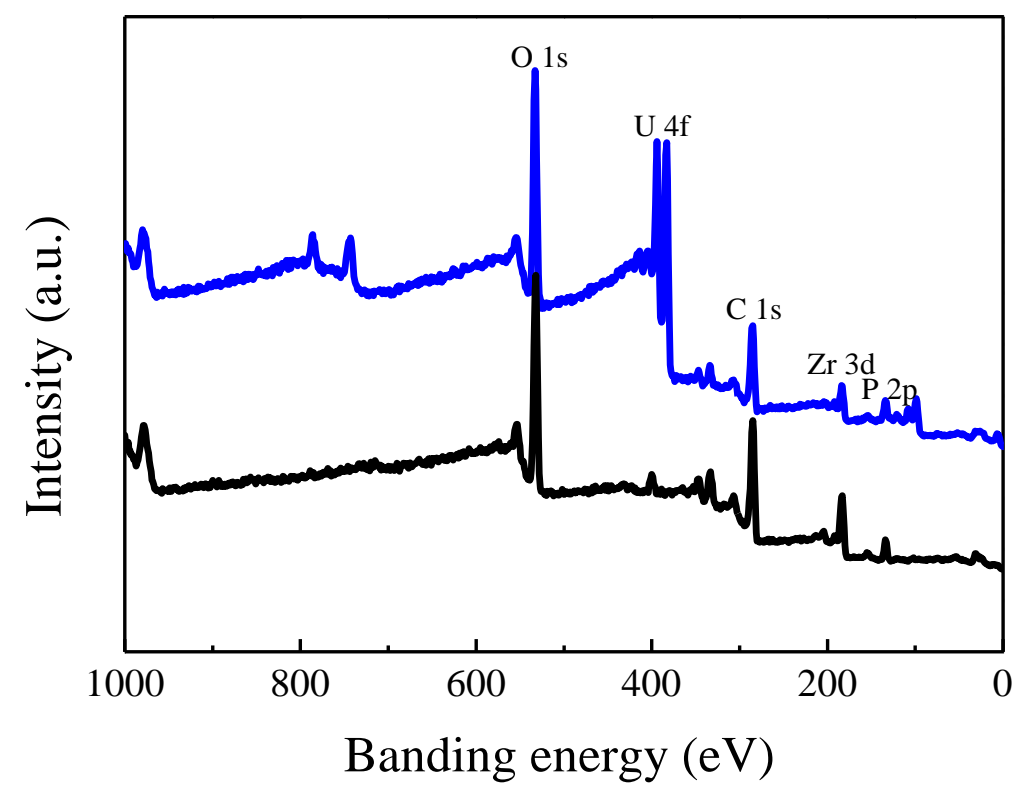

Figure S18 Wide-scan XPS spectra of PCN-222-PA (black) and U@PCN-222-PA (blue).

Table S1 Kinetics model parameters of U(VI) adsorption on PCN-222-PA.

\begin{tabular}{|c|c|c|c|c|c|}
\hline \multicolumn{3}{|c|}{ Pseudo-first-order model } & \multicolumn{3}{|c|}{ Pseudo-second-order model } \\
\hline$q_{e} / \mathrm{mg} \cdot \mathrm{g}^{-1}$ & $k_{1} / \min ^{-1}$ & $\mathrm{R}^{2}$ & $q_{e} / \mathrm{mg} \cdot \mathrm{g}^{-1}$ & $k_{2} / \mathrm{g} \cdot \mathrm{mg}^{-1} \cdot \mathrm{min}^{-1}$ & $\mathrm{R}^{2}$ \\
\hline 2.14 & 0.0091 & 0.786 & 10.01 & 0.024 & 0.999 \\
\hline
\end{tabular}

Table S2 Comparison of U(VI) maximum uptake capacity of PCN-222-PA with phosphorus-modified MOF adsorbents.

\begin{tabular}{ccc}
\hline Adsorbents & $\mathrm{q}_{\mathrm{e}} / \mathrm{mg} \cdot \mathrm{g}^{-1}$ & Refs \\
\hline $\mathrm{SZ}-2$ & 58.2 & $\begin{array}{c}\text { Nat. } \\
\text { Commun., }\end{array}$
\end{tabular}




\begin{tabular}{|c|c|c|}
\hline & & $2017,8,15369$ \\
\hline CMPO@MIL-101 & 22 & $\begin{array}{c}\text { Anal. Chem. } \\
\text { 2017, 89, 11, } \\
5678-5682\end{array}$ \\
\hline MIL-101-Ship & 28.0 & $\begin{array}{l}\text { J. Hazard. } \\
\text { Mater., 2017, } \\
\text { 335, 1-9 } \\
\text { J. Ind. Eng. }\end{array}$ \\
\hline $\mathrm{GO} / \mathrm{Fe}_{3} \mathrm{O}_{4} / \mathrm{OPO}_{3} \mathrm{H}_{2} / \mathrm{PCN}-222$ & 416.7 & $\begin{array}{c}\text { Chem., 2021, } \\
93,322-332\end{array}$ \\
\hline 808-MPA100 & 146.7 & $\begin{array}{c}\text { ACS Appl. } \\
\text { Mater. } \\
\text { Interfaces 2019, } \\
11, \\
\text { 33931-33940 }\end{array}$ \\
\hline PN-PCN-222 & $\begin{array}{c}303.5 \\
\text { (without } \\
\text { irradiation) }\end{array}$ & $\begin{array}{l}\text { Appl. Catal. } \\
\text { B-Environ., } \\
\text { 2019, 254, } \\
\text { 47-54 }\end{array}$ \\
\hline Phosphorylurea-UIO-68 & 217 & $\begin{array}{l}\text { Chem. Sci., } \\
\text { 2013, 4, } \\
\text { 2396-2402 }\end{array}$ \\
\hline PCN-222-PA & 401.6 & This work \\
\hline
\end{tabular}

Table S3 Comparison of U(VI) maximum uptake capacity of PCN-222-PA with various MOF adsorbents.

\begin{tabular}{ccc}
\hline Adsorbents & $\mathrm{q}_{\mathrm{e}} / \mathrm{mg} \cdot \mathrm{g}^{-1}$ & Refs \\
\hline \multirow{2}{*}{ MOF-76 } & & Chem. \\
& 298 & Commun., \\
& & 2013,49, \\
& & 10415-10417 \\
& & J. Radioanal. \\
& & Nucl. Ch., \\
UiO-66-NH 2 & 114.9 & 2016,307, \\
& & $269-276$ \\
& & Dalton. \\
MIL-101(Al) & & Trans., 2017, \\
& 110.0 & 46, \\
& & 12010-12014
\end{tabular}


Co-SLUG-35

$\mathrm{Zn}(\mathrm{HBTC})(\mathrm{L})\left(\mathrm{H}_{2} \mathrm{O}\right)_{2}$

AZO-MOFs

JXNU-4

MIL-101-DETA

Coumarin-modified

Zn-MOF-74

ED-MIL-101(Cr)

HKUST-1

$\mathrm{Fe}_{3} \mathrm{O}_{4} @ \mathrm{ZIF}-8$

PCN-222-PA
118

Chem. Eng.

J., 2017, 316,

154-159

J. Mater.

115 Chem. A, 2015,

3, 13724-13730

J. Radioanal.

Nucl. Ch.,

312.3 2016, 310,

353-362

Eur. J. Inorg.

129.3 Chem., 2019,

735-739

ACS Appl.

Mater.

314 Interfaces,

2016, 8,

31032-31041

J. Mater.

350.0 Chem. A, 2015,

$3,525-534$

J. Hazard.

$360.0 \quad$ Mater., 2016,

311, 30-36

200.0

Sci. Rep., 2015, 5, 13514

ACS Appl.

Mater.

2.68 Interfaces 2017, 9,38 , 32446-32451

Colloid

744 (298 Surface A, 2013, 431,

K) 87-92

Chem. Eng.

539.7 J., 2019, 378,

122105

401.6 This work 
Table S4 Thermodynamic parameters of U(VI) adsorption over PCN-222-PA with different temperatures.

\begin{tabular}{cccc}
\hline $\mathrm{T} /{ }^{\circ} \mathrm{C}$ & $\Delta \mathrm{G} / \mathrm{kJ} \mathrm{mol}^{-1}$ & $\Delta \mathrm{H} / \mathrm{kJ} \mathrm{mol}^{-1}$ & $\Delta \mathrm{S} / \mathrm{J} \mathrm{mol}^{-1} \mathrm{~K}^{-1}$ \\
\hline 298 & -18.2 & 19.7 & 127.2 \\
308 & -19.5 & & \\
318 & -20.7 & & \\
\hline
\end{tabular}

Table S5 The selectivity coefficient of U(VI) for different metal ions

\begin{tabular}{lllllllllllll}
\hline Metal & $\mathrm{Co}^{2+}$ & $\mathrm{Pb}^{2+}$ & $\mathrm{Zn}^{2+}$ & $\mathrm{Ce}^{3+}$ & $\mathrm{Dy}^{3+}$ & $\mathrm{Er}^{3+}$ & $\mathrm{Eu}^{3+}$ & $\mathrm{Gd}^{3+}$ & $\mathrm{La}^{3+}$ & $\mathrm{Nd}^{3+}$ & $\mathrm{Yb}^{3+}$ \\
\hline SU/M & 23.2 & 12.8 & 8.6 & 20.4 & 41.6 & 24.0 & 31.3 & 21.1 & 16.7 & 56.1 & 116.5 \\
\hline
\end{tabular}

\section{Kinetic models of U(VI) adsorption on PCN-222-PA}

Adsorption pseudo-first-order kinetic model and pseudo-second-order kinetic model is described as following:

$$
\begin{aligned}
& \ln \left(q_{e}-q_{t}\right)=\ln q_{e}-k_{1} t \\
& \frac{t}{q_{t}}=\frac{t}{q_{e}}+\frac{1}{k_{2} q_{e}^{2}}
\end{aligned}
$$

Where $\mathrm{q}_{\mathrm{t}}\left(\mathrm{mg} \cdot \mathrm{g}^{-1}\right)$ represents the adsorbed amount of the PCN-PA-222 at time $\mathrm{t}$ (min), $\mathrm{q}_{\mathrm{e}}\left(\mathrm{mg} \cdot \mathrm{g}^{-1}\right)$ is the uptake of adsorbate at equilibrium, $\mathrm{k}_{1}\left(\mathrm{~min}^{-1}\right)$ and $\mathrm{k}_{2}$ $\left(\mathrm{g} \cdot \mathrm{mg}^{-1} \cdot \mathrm{min}^{-1}\right)$ are the pseudo-first-order rate constant and pseudo-second-order rate constant, respectively. 


\section{Isotherm models of $\mathrm{U}(\mathrm{VI})$ adsorption on PCN-222-PA}

Adsorption Langmuir isotherm model is described as following:

$$
\frac{C_{e}}{q_{e}}=\frac{1}{k_{L} q_{m}}+\frac{C_{e}}{q_{m}}
$$

Where $\mathrm{q}_{\mathrm{m}}\left(\mathrm{mg} \cdot \mathrm{g}^{-1}\right)$ is the maximum adsorbed amount, $\mathrm{k}_{\mathrm{L}}$ is the Langmuir constant $\left(\mathrm{L} \cdot \mathrm{mg}^{-1}\right)$.

\section{Distribution coefficient and selectivity coefficient of $U(V I)$ adsorption} on PCN-222-PA

The distribution coefficient $\left(\mathrm{K}_{\mathrm{d}}, \mathrm{L} \cdot \mathrm{g}^{-1}\right)$ and selectivity coefficient $\left(\mathrm{S}_{\mathrm{U} / \mathrm{M}}\right)$ were calculated by the following equation:

$$
\begin{gathered}
K_{d}=\frac{\left(C_{0}-C_{e}\right) V}{C_{e} \cdot M} \\
S_{U / M}=\frac{K_{d}^{U}}{K_{d}^{M}}
\end{gathered}
$$

Where $\mathrm{C}_{0}\left(\mathrm{mg} \cdot \mathrm{L}^{-1}\right)$ is the initial concentration of metal ion, $\mathrm{C}_{\mathrm{e}}\left(\mathrm{mg} \cdot \mathrm{L}^{-1}\right)$ is the equilibrium concentration of corresponding metal ion, V (L) is the volume of solution, $\mathrm{M}(\mathrm{g})$ is the weight of PCN-222-PA. 